\title{
User Experience Design for Mixed Reality: A Case Study of HoloLens in Museum
}

\begin{abstract}
In recent years the applications of Mixed Reality (MR) processing has become highly apparent in academia and the manufacturing industry with the release of innovative technologies such as the Microsoft HoloLens. However, crucial design issues with the HoloLens' restricted Field Of View (FOV) to a narrow window of 34 degrees inhibited the user's natural peripheral vision (Kress $\&$ Cummings, 2017). This visual limitation results in a loss of pre-set functions and projected visualisations in the AR application window. This paper presents an innovative methodology in designing a spatial User Interface (UI), to minimise the adverse effects associated with the HoloLens' narrow FOV. The spatial UI is a crucial element towards developing a museum-based MR system, which was evaluated by nine experts in Human-Computer interaction (HCI), visual communication and museum studies. Results of this study indicate a positive user reaction towards the accessibility of the spatial UI system and enhancing the user experience. This approach can help current and future HoloLens developers to extend their application functions without visual restrictions and missing content.
\end{abstract}

Keywords: Microsoft HoloLens, Field of View, User Experience, Usability, Mixed Reality, Spatial UI.

\section{Introduction}

Mixed Reality and immersive technologies are highly discussed topics in modern marketing strategies (Spreer \& Kallweit, 2014) and are considered one of the most sustainable marketing tools available today (Bulearca \& Tamarjan, 2010). Furthermore, the manufacturing cost of wearable devices has reduced substantially making them financially viable to most individuals and small companies (Yang, $\mathrm{Yu}, \mathrm{Zo}, \&$ Choi, 2016). Many multinational companies have invested in Augmented Reality (AR) and Virtual Reality (VR) hardware and software such as Microsoft, Apple and Google (Evans, Miller, Pena, MacAllister, \& Winer, 2017) and in particular, devices that provide immersive mixed reality UX (Kalantari \& Rauschnabel, 2018).

Market research predicts that 411 million smart devices will be sold by the year 2020, and the shipments in AR and VR headsets will increase substantially to 96 million devices by 2020 (Insight, 2016). Wireless MR Head Mounted Displays (HMD) have extended the use and applications of virtualisation technologies. The inauguration of MR HMD technologies in the public domain has enhanced peoples wellbeing, conceptualisation and personal lifestyle (Kalantari, 2017). These wearable technologies are designed to interconnect and allow simultaneous realtime communication and information retrieval (Park, Chung, \& Jayaraman, 2014). The primary function of these devices is to amalgamate the real world and a virtual 
spectrum into a singular viewpoint by changing the user's perception of the real environment (Rauschnabel, 2018).

The advantage of AR over other forms of virtual visualisation is that it enhances the user's experience in the real environment instead of replacing it (Chuah, 2018). Recent studies indicate that consumers are using wearable AR technologies more frequently as they are becoming more accessible and user-friendly (Kalantari \& Rauschnabel, 2018). Moreover, several studies were conducted to enhance the accessibility of wearable devices' and functions for the inexperienced user (Tom Dieck, Jung, Han, \& Technology, 2016).

A well-known taxonomy named 'M502' coined by Milgram, Takemura, Utsumi, and Kishino (1994) defines MR among other technologies such as VR. The model states that MR is a technology that consists of amalgamating 3D content and the real-world environment. In contrast, VR is a single virtual environment isolated from the real world. (Azuma, 1997). In recent years new devices have emerged and changed the concept of MR applications. A new taxonomy created by Bray (2018), redefines the capabilities of the MR headset. These new models incorporate improved sensors and the capability to communicate with multiple users in the same environment simultaneously and change the relationship between the physical and virtual environment in MR technologies, such as spatial mapping for Microsoft HoloLens (Zeller, Baker, \& Bray, 2018b), and The Mirror World (Ricci, Piunti, Tummolini, \& Castelfranchi, 2015).

Therefore, MR is merely a form of VR with expanded capabilities and the incorporation of real-world environments. Figure 1 depicts (Bray's, 2018) continuum of MR 'Holographic devices', which comprises of all devices that have different capabilities to immerse users in MR environments. These holographic devices vary according to the level of user immersion, for example, MR technologies such as Vuzix, ODG (Charara, 2017), Epson Moverio (Epson, 2015), and Google Glasses (Inc., 2014) have restrictive and limited interactivity between the virtual and actual environment in comparison to MR technologies such as Microsoft HoloLens (Microsoft, 2015), Magic Leap (Leap, 2018), Meta 2 (Prasuethsut, 2016) that allow a greater natural interaction between the virtual content and the natural environment.

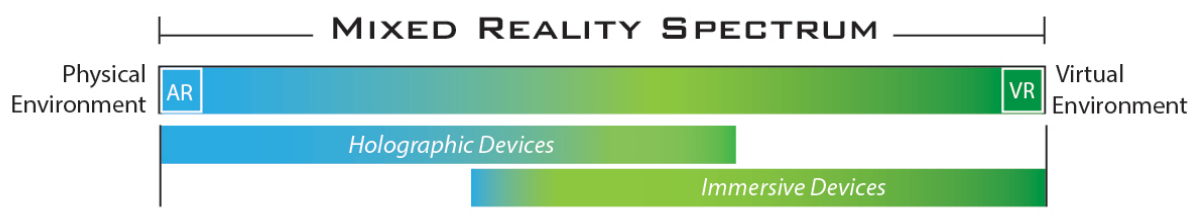

Fig. 1 Mixed Reality concept by (Bray, 2018) and allocation of Holographic and immersive devices

MR can change the user's perception of the real environment (Rauschnabel, 2018). It also enhances the user's experience in the real environment instead of replacing it (Chuah, 2018). The advantage of using MR headsets is that it allows users to 
walk freely within the MR environment (Evans et al., 2017). The vision-based wearable devices concept further enables users to interact in the MR environment using hand gesture controls ( $\mathrm{Lv}$ et al., 2015). Hardware limitations and restricted image processing inherent in standard MR HMDS produces instability and discomfort for many users (Hsieh, Jylhä, Orso, Gamberini, \& Jacucci, 2016). However, the Microsoft HoloLens is unique amongst other MR with its ability to explore MR environments hands-free using only gesture control and position mapping (Evans et al., 2017). This technological advancement allows unrestricted user mobility in comparison to HMDs that require wired external controllers and large components. Furthermore, this approach provides content registration through spatial mapping techniques (Coppens, 2017).

These accessibility features offer a wide range of application development opportunities and research explorations into User Experience (UX) of the Microsoft HoloLens HMD. Virtual Reality (VR) applications are examples of other spatial 3D user interfaces (LaViola Jr, 2008). However, in non-see-through VR HMDs, the user cannot perceive their actual physical surroundings (D. Bowman, Kruijff, LaViola Jr, \& Poupyrev, 2004). An advantage of implementing spatial MR UIs over the standardised VR digitised screen is that MR is visually less-restricted than the physical boundaries of the VR screen. Furthermore, spatial MR UI permits users to freely explore open spaces (Billinghurst, Poupyrev, Kato, $\&$ May, 2000). The spatial mapping feature of the HoloLens is suitable for creating MR UI for location-aware applications (Höllerer, Feiner, Terauchi, Rashid, \& Hallaway, 1999). The standard HoloLens UI acts as a virtual spectrum between the user and the physical environment. Therefore, MR HMDs like the HoloLens is more effective than VR systems for developing interactive spatial UI environments. Collaborative visual interfacing across the HoloLens platform allows simultaneous group interactions and engagements to create shared visceral experiences. However, a critical factor in enhancing the user's natural ability to interact within the MR environment concerns the measuring of distance travelled, the manipulation of objects and other environmental factors such as the navigation of physical obstacles (Bowman et al., 2004).

The cognitive and physical abilities of the MR user may become impaired due to physical limitations, such as arm length which can cause issues with the gesture control of the MR HMDs. These issues may also impact the frame-rate of the MR system to operate effectively in real-time (Bowman \& Hodges, 1999). Microsoft HoloLens has been reviewed frequently from developers and scholars from different aspects (Evans et al., 2017) (Zhang, Dong, \& El Saddik) (Chuah, 2018) since release. Although the HoloLens has many positive aspects that make it distinctive over other MR HMD, it has significant technical problems. One of the significant issues of the HoloLens is its limited FOV for which impacts system usability and UX. According to Kress and Cummings (2017), the HoloLens FOV is 34 degrees; however, Keighrey, Flynn, Murray, and Murray (2017) stated the FOV as 30 degrees. Hockett and Ingleby (2016) suggest that HoloLens viewport 
is not suitable for the average user's peripheral vision. As the average person's natural FOV is $130^{\circ}-135^{\circ}$ vertically (Dohse, 2007) and $200-220^{\circ}$ horizontally (Szinte \& Cavanagh, 2012) and the HoloLens' peripheral vision according to Keighrey et al. (2017) is $30^{\circ}$ by $17.5^{\circ}$. Milgram et al. (1994) further suggest such visual limitations in the FOV conflicts with Milgram's theorem about the minimum FOV for see-through displays to operate effectively. The limitation of the HoloLens visual capacity is a hindrance for many developers to create panoramic effects. The optical accessibility issue of the HoloLens affected the level of user immersive experience negatively (Bright, 2015) and diverted the attention (Hockett \& Ingleby, 2016).

\subsection{Related Work}

Usability is defined as 'ease of use' in addition to 'usefulness', and this can include the learnability aspect, accuracy and speed of performing tasks, error rates, and users satisfaction (Hix \& Hartson, 1993; Shneiderman \& Plaisant, 1992). Similar studies in usability in MR systems such as (Lee, Nelles, Billinghurst, \& Kim, 2004) (Paelke, Sester, \& Sensing, 2010) and devices (Wagner, Pintaric, Ledermann, \& Schmalstieg, 2005) (Bach \& Scapin, 2004) explore the criteria of evaluating the MR systems such as the ergonomic factors which comprise of UI, real and virtual environment and the fusion of elements.

Another study surveyed different evaluation methods for usability of virtual environments (D. A. Bowman, Gabbard, Hix, \& Environments, 2002). Scholars introduced a framework for measuring usability through evaluating the ergonomic factors for interactive MR devices (Bordegoni, Cugini, Caruso, Polistina, \& Manufacturing, 2009).

However, very few software developers have utilised the application development capabilities of the HoloLens. A potential reason for this may be the high price of the HoloLens HMD unit in comparison to other MR devices. However, despite the cost, many innovative applications have been developed for use in modern industry. For example, HoloMuse is an MR application for individuals to engage with archaeological artefacts through gesture-based interactions (Pollalis, Fahnbulleh, Tynes, \& Shaer, 2017). Another application was developed to provide an in-situ personal assistant for user's (Blattgerste, Strenge, Renner, Pfeiffer, \& Essig, 2017). The HoloLens HMD was also adapted to incorporate a finger-worn camera to provide magnification for sufferers of poor vision (Stearns, DeSouza, Yin, Findlater, \& Froehlich, 2017). Moreover, the HoloLens has contributed to the medical field and evolved 2D graphics into 3D interactive visuals using MR technologies (Syed, Zakaria, \& Lozanoff, 2017). A similar adaptation was implemented to aid visual precision in medical surgeries (Pratt et al., 2018) (Adabi et al., 2017). Other notable uses of the HoloLens HMD include; the prototyping of designs (DeLaOsa, 2017), gaming applications (Vople 2015; Alvarez 2015).

Tourism and cultural heritage witnessed the incorporation of MR technology in the touristic experience and its influence on the visitor was evident. MR was able to extend the archaeological sites in the 'SHAPE' project in order to enhance the 
educational and social experience for the visitors (Hall et al., 2001). Another study 'Dinohunter' used the MR in for the sake of learning, entertaining and discovering (Sauer \& Göbel, 2003). Moreover, MR had the capability to enhance the visual experience by extending the exhibition space with virtual content (Hughes, Smith, Stapleton, \& Hughes, 2004). It was also adopted to encourage communication and disseminating knowledge (Brondi, Carrozzino, Lorenzini, \& Tecchia, 2016).

The existence of Microsoft HoloLens was apparent in tourism, museums and cultural heritage projects (Raptis, Fidas, \& Avouris, 2017), (Cortana, 2017). The 'HoloMuse' application engages users with archaeological artefacts through gesture-based interactions (Pollalis et al., 2017). HoloLens has contributed to restoration in art galleries by adding a virtual extension of antiques (Melnick, 2017). Recently, HoloMuseum emerged as a management tool to explore the virtual extension of the exhibited antiques in museums (Bottino, García, \& Occhipinti, 2017).

However, many users have complained about the limitations of the HoloLens' FOV causing the disappearance of displayed content from the interface. This issue was particularly prevalent during the creation of the Heritage Building Information Modelling project (Fonnet, Alves, Sousa, Guevara, \& Magalhães, 2017) and the Holo3DGIS application that suggests a similar development issue with the inability to display full user content further supports this problem (Wang, $\mathrm{Wu}$, Chen, \& Chen, 2018). Therefore, designing a customised UI for HoloLens applications is a viable solution for enhancing UX. Available resources concerning UI in see-through MR HMD optics and its guidelines is limited (Evans et al., 2017), this lack of research has led to challenges when developing UI and UX software.

Existing literature relating to customised UI design for mixed reality applications using headsets is limited (Evans et al., 2017) and most of these works were written by developers to communicate practical issues and guidelines in implementation rather than user experience design. Due to these resource restrictions, HoloLens application developers need a clear guidance for enhancing usability and UX. This literature review identifies two primary gaps in current research concerning spatial UI and UX. They are: 1. A method is required to redesign and restructure the spatial user interface to expand the current limitations of the HoloLens FOV. 2. Further research into UI methods concerning the major outside factors; user experience, environmental considerations, user characteristics and system aspects as outlined in Bowman \& Hodges (1999).

We developed a spatial UI design for HoloLens applications that overcomes the limitation of HoloLens' narrow FOV. This spatial UI is part of a prototype built for guiding museum visitors using a virtual guide in MR that allow interaction between the visitor and virtual content in the real environment. The hypothesis of this research is that the spatial UI following user experience design principles improves the usability of HoloLens-based mixed reality. Finally, an evaluation of this prototype has been conducted to validate the design. 


\section{System Overview}

The purpose of the MR application developed in this project is to permit a personal interactive virtual museum tour guide UI to assist navigation and storytelling. This process includes a virtual overlay of physical exhibits within a museum that can be manipulated in the MR spatial UI by the user. This prototype is developed specifically for testing a spatial UI for cultural heritage guidance UX, to test the research hypothesis outlined in the literature search. This procedure aims to create a simple, interactive and informative guidance framework for museum patrons to use. The MR application requires the user to wear a Microsoft HoloLens and explore a series of virtual content through the new spatial UI interface. An environment populated by museum artefacts is required to superimpose digital virtualisations and information for the virtual tour guidance system to operate.

\subsection{Functionality}

The objective of this study is the develop an MR UI that has the visual capacity to achieve maximum user interactivity. The test procedure outlines controlled user interactions with spatial 3D models, images, videos and buttons. These operations include:

1. Hand gesture control of floating virtual replications of museum artefacts to rotate them 360 degrees on a central pivot using finger dragging gestures.

2. Buttons that initiate text and images for receiving information.

3. Engaging with the virtual character guidance system that explains audio and visual information in real-time and also replaying that information

4. Utilising small air click prompted circles that work as trigger objects to reveal information about particular areas of interest.

\subsection{System Architecture}

The system framework for the MR was created using three developmental phases as depicted in Fig 2.

1. Assets creation: To develop a contemporary and engaging spatial UI AR application infrastructure for the HoloLens a 2D design was created using Adobe Photoshop and Illustrator software. Graphical content was exported in Portable Network Graphics (PNG) and Joint Photographics Experts Group (JPEG) image formats to import as texture-based assets. The 3D elements of the application were custom sculptured using ZBrush and modelled using Autodesk Maya computer software. Marvellous Designer also was adopted to create realistic clothes for virtual characters. Substance Painter was used for finishing the surficial elements of the 3D models to provide further depth and detail. 3D scanning technology was employed to replicated physical objects using the mobile phone software 123D Catch and also using the Cubify $3 \mathrm{D}$ handheld scanner. The image then went through a refinement process in Recap 360. Finally, the anatomical elements were produced manually and with using Perception Neuron motion capture suit. 
The facial animation was performed manually via a facial capture system using a Kinect camera to transfer movement and refine animations. The first phase resulted in a considerable number of file formats for utilisation across different platforms. The 3D assets produced in FilmBox (.FBX) property files, which contain positional data are utilised alongside PNG and JPEG image files to construct the first visualisations for the UI design infrastructure. Also, the 3D animation files will be in (.FBX) format. These are incorporated with audio rendering and sound effect libraries to create the finished effect.

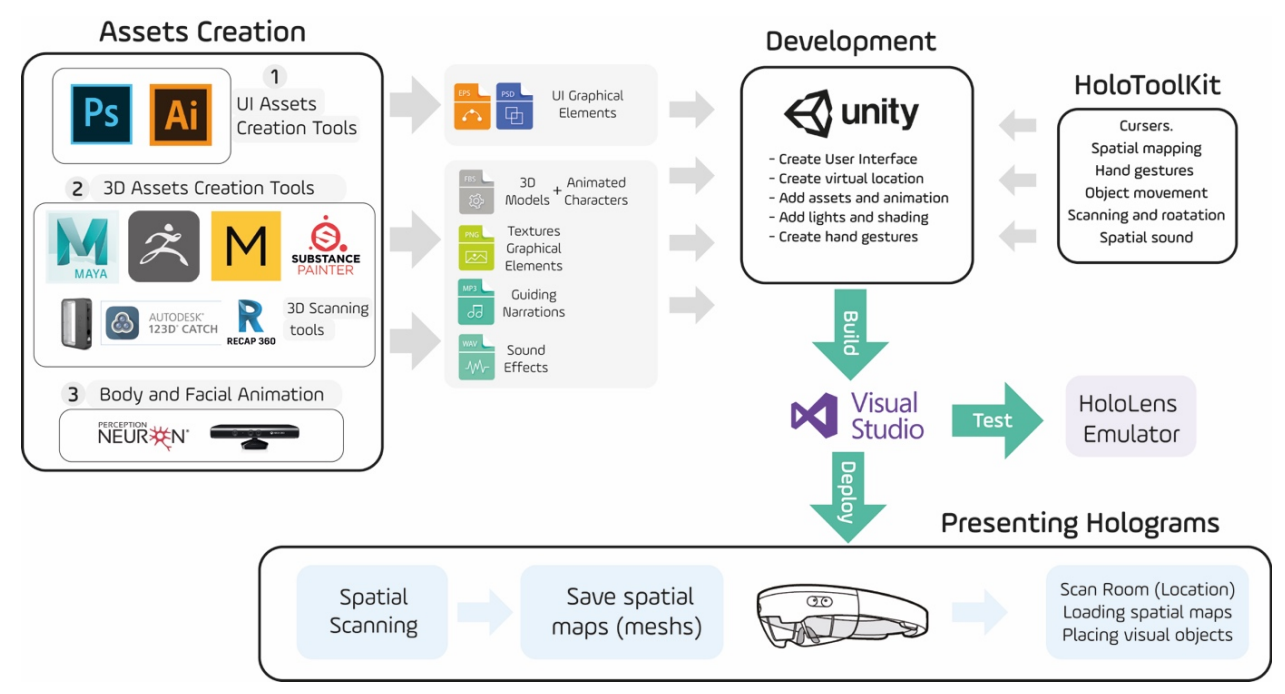

Fig 2. MR System Architecture

2. Developmental Procedure: HoloToolKit was employed to develop the system using the toolkit that comprises scripts and components that facilitate building the intended functionalities in HoloLens (Microsoft, 2017). The toolkit has a component of cursors, spatial mapping and understanding the physical environment, hand gestures, object movement functionalities, scanning and rotation functionalities, and spatial sound. Mainly, the developments practically conducted in Unity 3D as it accommodates all imported files in the scene and also the toolkit mentioned is imported to build the development functions planned. The development can be broken down into creating a central gaze position for the user to control functions naturally within the application, so the screen is positioned in front of the user at an appropriate distance and examining the spatial mapping of the physical environment to reallocate the UI guide system location monitor next to the actual physical object. The MR application is designed to respond to the operator hand gestures for handsfree interactions to control the orientation of 3D scanned objects. The process continues by placing all characters, props and virtual antiques in the scene, then add the character animation with consideration to be centralised around the user. The scene creation ends by adding lights and dropped shadows to enhance the blending between the virtual and the physical environment. 
3. Compiling and Deploying the Application: The application deployment starts by building the application and import it into Microsoft Visual Studio which deploys the virtual environment developed in Unity 3D into the HoloLens utility through USB data transfer, and this procedure completes the design process. Some MR developers prefer to test and simulate the running application on the device in the 'HoloLens Emulator' to shorten the time of the unexpected amendments and fixing bugs.

Presenting holograms in the physical environment depend on the process of spatial mapping, and this process starts when the HoloLens begins to scan the environment during start-up. It saves the scanned environments in its library and loads the virtual overlay it once it recognises a room or environment. This spatial scanning comprises of mesh or polygons that represent a detail representation of the real world, and it is created on top of the physical environment to represent it in the MR scene, as depicted in Fig 3 (Zeller et al., 2018b). Therefore, this mesh is the critical element of the mixed reality visualisation as it encompasses real objects with a virtual overlay to create a single visual spectrum.

Upon opening the application in the HoloLens, the user can perform the required hand gestures to instigate an interaction with the virtual environment and initiate the virtual tour guide system.

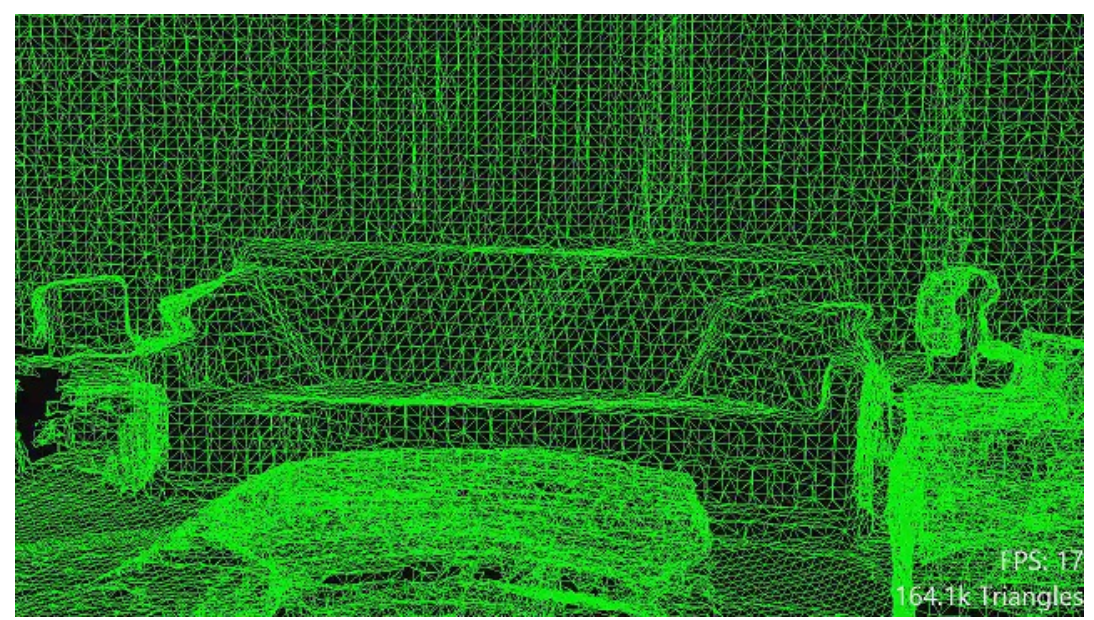

Fig 3. Spatial mapping created by HoloLens sensors, source: (Zeller, Baker, \& Bray, 2018b)

\subsection{UI Design Process}

To develop a spatial UI design framework for the HoloLens requires the curvature of the visuals that surround the user. This approach allows the position of interactive points closer to the user to ease in-application interactions. Fig 4, demonstrates the user surroundings are utilising brightened areas of the screen to highlight areas of interaction while the darkened areas give ambience and depth to invisible areas of the visualisation. However, the darkened areas represent the real 
environment without imprinted virtual content. Furthermore, the issue of FOV restriction is observable, and the areas outside of the highlighted square indicate the missing onscreen content.

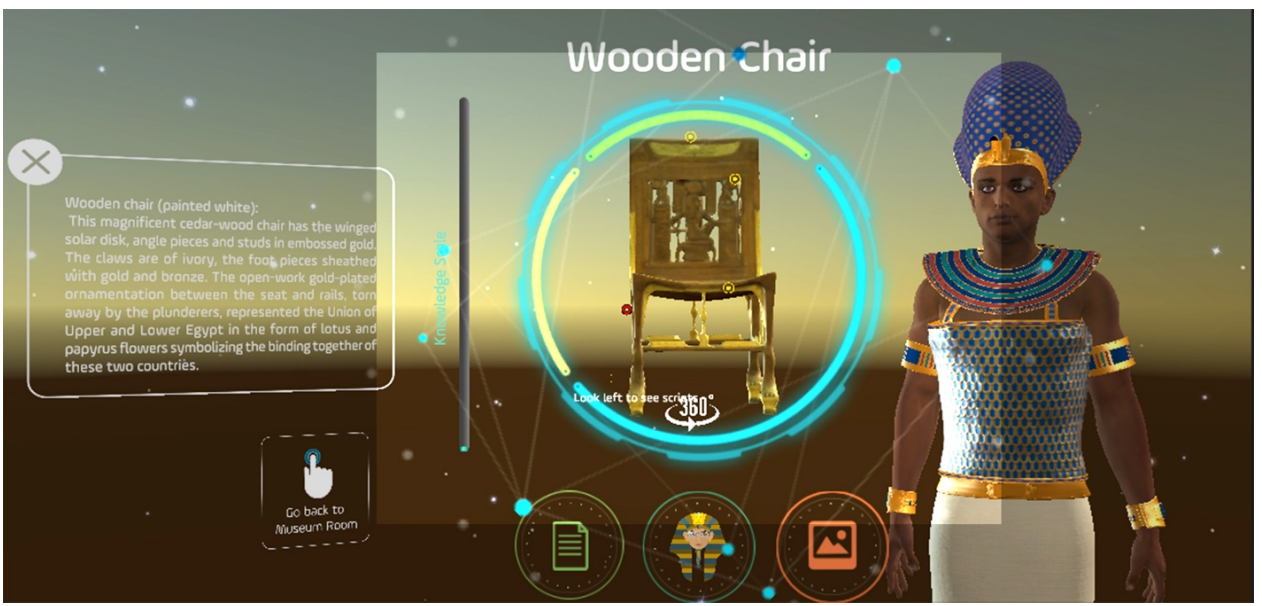

Fig 4. Spatial UI Design as if the user can see it from HoloLens

Due to the limitations of the HoloLens FOV, the virtual environment resulted in clipping the image as shown in Fig 5. The outcome of this spatial issue prohibits users from observing additional content to the left and right of the central screen partition. To examine this virtual environmental irregularity in detail, a series of experiments adapted from Bowman and Hodges (1999) study into the influence of external factors.
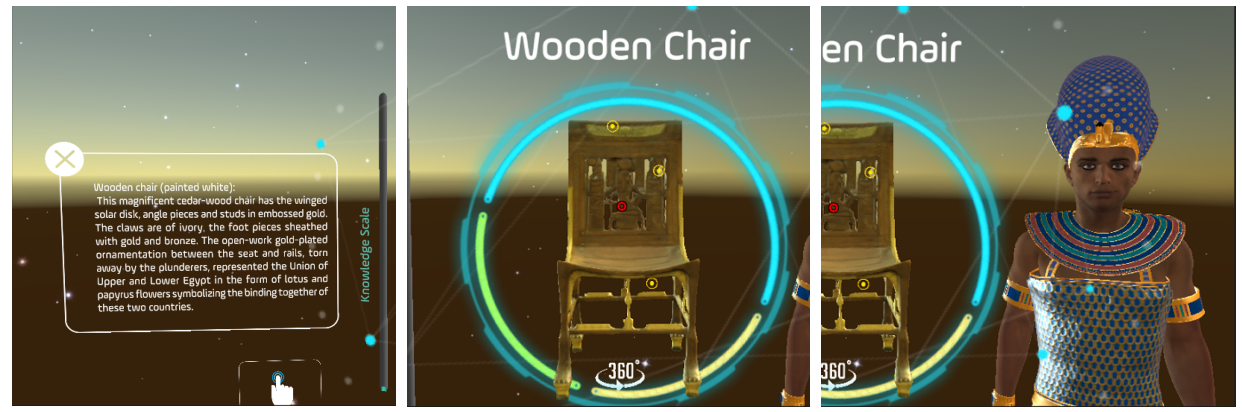

Fig 5. Cropped Scene from the perspective of the HoloLens User

However, external factors affect the spatial screen surroundings outside of Bowman \& Hodges theory that propagated interesting results. Examining and testing potential solutions to these issues produced a spatial UI format that had the potential to enhance the accessibility and interactivity of the application environment. The UX principles of creating spatial UI design offer a solution for the HoloLens shown in Fig 6. 
Ramy Hammady, Minhua Ma \& Carl Strathearn

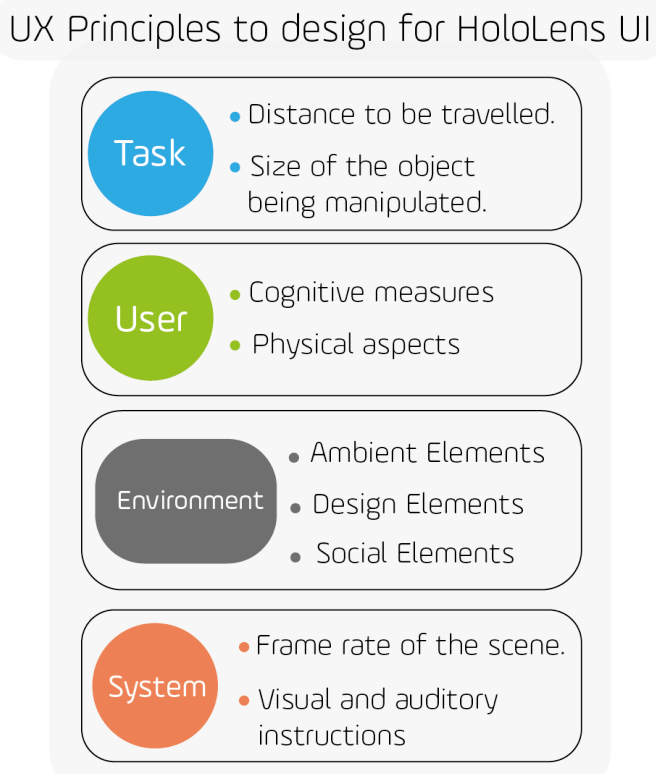

Fig 6. UX principles for HoloLens UI design adapted from (Bowman \& Hodges, 1999).

1. Task: As outlined in (Bowman \& Hodges, 1999) the characteristics of a task is the representation of all external aspects the influence performance. In the spatial UI prototype, the user is required to walk to the UI and move the head then point with the hand at the centre of the gaze panel then perform air-tap gestures. The user is required to walk around and observe physical artefacts while performing these tasks to initiate interactions in a controlled environment. User accessibility is critical as a test procedure since users are required to drag objects and utilise gestures in the application to manipulate the virtual environment. However, to assess the practicalities of these controls, some calculations have to be accounted:

a: Distance and Travel: Limitations of the HoloLens FOV effects what the user can observe this accounts for around a quarter of the available screen capacity. Furthermore, the user is required to observe a physical object simultaneously with the virtual UI. Calibrating the distance of the UI indicated that an optimal range of 1 meter is required to perform gesture-based interactions easily. However, the user needs to be 2.2 meters away to see the whole screen (Fig 7). This distance resulted in an instability in the control function that rotates virtual artefacts. The first test focused on control of scene triggers at a distance of 1 meter; the results of this process prohibited the entire FOV of the user. In an attempt to resolve the narrow spatial UI vision issue, voice command instructions prompted the user to look left or right. This process preserved the screen triggers more accurately than previously as the view range is 2.2 metres from the exhibited object. However, this altered the depth of the UI which resulted in triggers outside of the desired locations, yet, the users retained a full field of vision unlike previously.

b: Size of the virtual objects: According to data gathered by participants in the experiment, the optimal size of the manipulated object for both comfort and 
functionality is $50 \mathrm{~cm} \times 50 \mathrm{~cm}$. Additionally, the maximum spatial UI distance is 1 -meter $\mathrm{x} 1$ meter. It is also important to state that participants managed to navigate the application and environment through gesture control with a minimum of external support from the experiment controller.
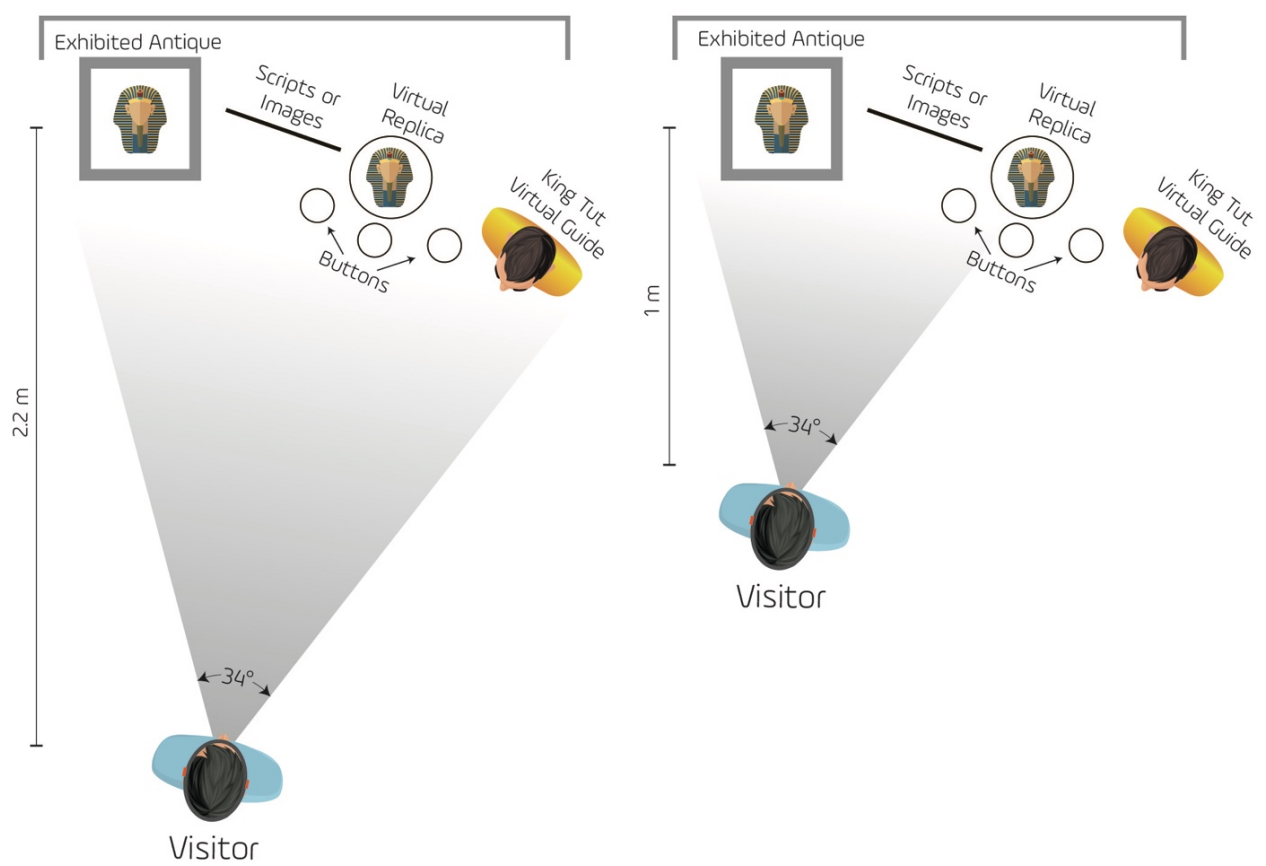

Fig. 7 Visualising the virtual spectrum at a variable distance

2. Environmental factors: To acquire an inclusive representation of the museum environments requirements for optimal mixed reality experience, Baker (1986) defined three components of the environment as follows: ambient elements, which are non-visual factors such as (temperatures, sounds, odours), design elements, which include visual factors such as (layout, colour, interior design), and social elements, which represent people such as (presence of visitors, peer-visitors). Some of the elements of the mentioned components are significant to be considered to achieve a better immersive experience:

a. Ambient elements: One of the most significant factors for presenting and visualising visuals is the environmental lighting conditions. Optimum lighting conditions for displays MR visualisations is low-light to dark conditions as the opacity becomes more apparent in interior lighting conditions. However, in bright areas of direct sun exposure causes virtual artefacts to lose their opaqueness. The museum setting provides a sufficient amount of low-light areas for the HoloLens to operate efficiently without losing image definition.

b. Design elements: To utilise the full spatial potential of the HoloLens virtual environment an adequate amount of space is required for the user to navigate 


\section{Ramy Hammady, Minhua Ma \& Carl Strathearn}

around the platform. Therefore, one of the factors that affect visualising holograms is the number of obstacles between the user and the augmented visuals. For the application to run effectively, the user has to have an open area in which to navigate around the virtual environment. Museum settings also should be considering the display spacing between exhibited items is designed to adopt wider space where can embrace the visitor wearing MR headset, space for holograms next to it and space for other visitors or peer-visitors.

c. Social elements: Peer-visitors interaction is beneficial especially if the mixed reality experience is sharing visuals and interaction between them. It even can enhance and enrich the museum experience. However, the only operational requirement may prove an issue in a museum setting is that people may walk in front of the HoloLens HMD or crowd around a specific exhibit. This optical process can cause deformation of the virtual mapping system against the physical environment.

3. User characteristics: The physical and cognitive abilities of the user to operate the HoloLens effectively.

a. Cognitive measures: The population sample utilised in this research study were given minimal instructions to control the application. However, during this phase, it became apparent that some participants required further instructions to operate the HoloLens HMD unaided. This issue relates to attaining the required skillset through practice using the HoloLens HMD to learn how to control the virtual environment.

b. Physical aspects: Variability of individuals heights within the test group provided interesting data regarding potential safety issues when utilising the HoloLens HMD. The optimal positioning of virtual elements within the application is for individuals that are approximately 1.7 meters in height. During the test procedure, people of smaller stature than 1.7 meters were observed looking up at the virtual objects. This angulation of the head may cause stress on the user's neck due to the weight of the HoloLens HMD. Over prolonged usage, this weight may become uncomfortable or potentially lead to injury. Comparatively, users taller than 1.7 meters had to look down at the visualisations causing similar strain on the neck. Scaling the spatial UI environment relative to the user's height provided a solution to this issue. This solution was achieved by calculating the distance from the camera to the ground within the application and scaling the UI based on the height of the operator reducing the risk of injury and discomfort.

4. System characteristics: All operational aspects regarding the hardware and software of the HoloLens.

a. Frame rate: Results of the experiment indicated that a drop in frame-rate to 15-20 frames per second was recorded during instances when many 3D visualisations appeared on the screen at once. Overloading the system may cause lagging of the application in some virtual environments that are heavily populated by $3 \mathrm{D}$ objects. In contrast, higher frames rates when observing single virtual artefacts produced higher levels of the objects surficial definition. Therefore, it is 
recommended that future developers of the HoloLens consider the spatial capacity of the virtual environment and populate this with artefacts at a distance from one another to reduce a drop in frame rate by positioning many $3 \mathrm{D}$ objects together on the screen at once.

b. Visual and audible instructions: From a user perspective it should be obvious that all interactive elements within the application are designed to be seen and heard. This process is enabled to engage the user fully to maintain attention and interest in the application. Losing content or not recognising audible instructions may lead to a reduction in the quality of the UX. To ensure the operator has a clear indication of the virtual environment and its interactive elements a 'tag along' methodology was employed to give the user visual prompts to achieve specific tasks as used in a study by Fonnet et al. (2017). This adaptation presents the user with sustained and accessible content. Audio prompts and instructions were used to compensate for the lack of visual information within certain unpopulated areas of the virtual space.

\section{Prototype Evaluation}

A field test of the new HoloLens spatial UI was conducted using a selective population sampling methodology to represent the average museum patron. The evaluation process examines the usability and assessability of the HoloLens HMD and spatial UI application during system usage. The testing of the spatial UI application and HoloLens within a public library setting is to examine the feasibility and application of this framework within a museum environment.

\subsection{Methodology}

The population sample of the HoloLens / spatial UI evaluation included nine experts in different academic disciplines ranging from; Human-Computer Interaction (HCI), Visual Communication and Museum Studies, as presented in Table 1. Considering the HCI and visual communication experts in this evaluation was made due to their abilities to assess -qualitatively and quantitatively - the usability, interactivity and the level of user experience gained. Then, the museum studies experts were considered to assess whether this system can achieve what museum visitors require in museums and map it according to the nature of museum visits. Considering experts to this evaluation has double benefits; their expertise as mentioned and they also can generally be museum visitors, so their responses can be more critical and beneficial to the study more than regular museum visitors. This approach is adapted from a previous study using a selective population sample of experts by Karoulis, Sylaiou, and White (2006), which yielded strong results utilising this data collection method.

The participants were asked to examine and evaluate the HoloLens and application based on their area of expertise. The evaluation mainly measures the usability aspect, and the user experience can be achieved. This approach is adapted from a 
previous study using a selective population sample of experts by Karoulis et al. (2006), which yielded strong results utilising this data collection method. Before the evaluation was conducted participants were given a short tutorial on the control functions of the HoloLens HMD, Fig. 8. The data collection technique was employed to allow both structured and open-ended enquiries in qualitatively manner (Labuschagne, 2003). The questions of the survey were designed based on the System Usability Scale (SUS) (Brooke, 1996), which is a dependable tool for measuring usability in different systems quantitively. This approach permitted the respondents to openly express opinions based on their area of expertise in certain areas, while also employing rating statistics such as the Likert Scale to gain precise data strings. Before the execution of the questionnaire, a pilot study was conducted to test the viability of the questions and the mixed data gathering approach. The time scale of the evaluation was approximately $5-10$ minutes per participant. The sampling selection of the study consisted of age group, the participant's area of expertise and previous experience.

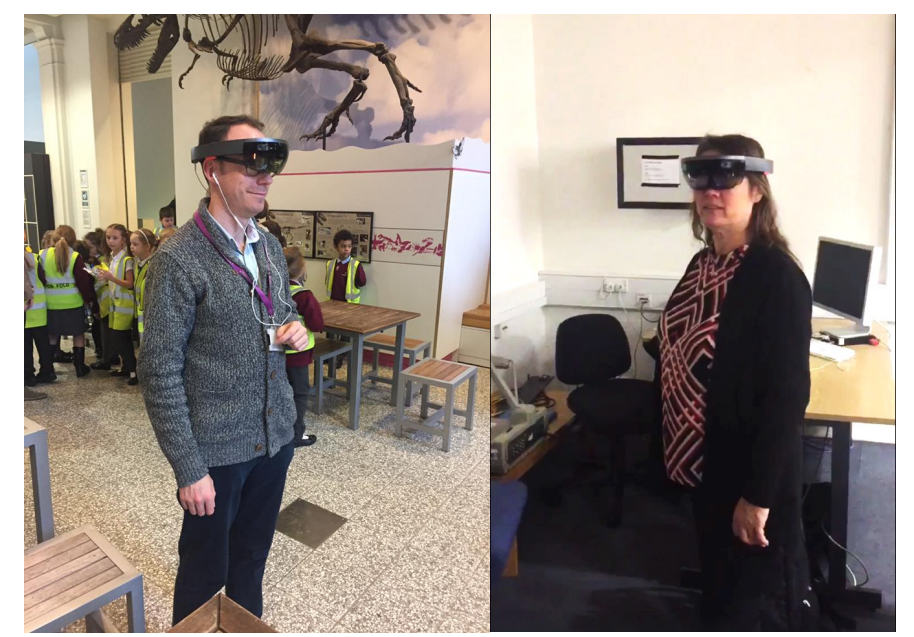

Fig. 8 Participants testing the HoloLens

\subsection{Results}

The numeric data represented in Table. 2 indicates the usability factors relating to UX, performance and functionality. This questionnaire is measured using the reliability factor of Cronbach's alpha $>0.65$.

The first inquiry of the questionnaire concerned the comfortability and convenience of wearing the HoloLens HMD. This area received the lowest user ratings 3.67 due to the weight of the HMD $(579 \mathrm{~g})$ putting a strain on the participant's neck. As one expert commented "It was a bit heavy on my neck, so I would not want it on too much longer. However, the vision and sound were 
fantastic". Moreover, the other two comments were "Not bad at all - Slightly heavy perhaps", and "Little bit heavy".

The second question examined the UX experience within the virtual environment in regards to significant issues such as a headache or dizziness when looking at the MR monitor during free motion. This question gained the highest mean values 4.78. Respondents indicated little to no disorientation when operating the HoloLens HMD. One participant remarked, "I felt immersed in the location without losing track of my surroundings; it was a good experience". Another expert noted, "The HoloLens was much better than VR headsets, there was no disorientation or loss of the horizon".

\begin{tabular}{|l|c|c|c|}
\hline \multicolumn{1}{|c|}{ Discipline expertise } & $\begin{array}{c}\text { Malel } \\
\text { Female }\end{array}$ & $\begin{array}{c}\text { Years of } \\
\text { Experience }\end{array}$ & Age Group \\
\hline $\begin{array}{l}\text { Academic and professional expert in Visual } \\
\text { communication and Arts }\end{array}$ & F & 22 & $45-60$ \\
\hline Expert at public engagement in museums & F & 7 & $31-45$ \\
\hline Expert in museum curatorship & M & 7 & $25-30$ \\
\hline Expert in museum curatorship & F & 6 & $31-45$ \\
\hline Expert in museum curatorship & F & 4 & $25-30$ \\
\hline Expert in HCl and visual interactions & F & 9 & $31-45$ \\
\hline $\begin{array}{l}\text { Data manager and responsible for enhancing the } \\
\text { museum visitor engagement }\end{array}$ & $\mathrm{F}$ & 2 & $31-45$ \\
\hline Expert in museum curatorship & $\mathrm{M}$ & 10 & $31-45$ \\
\hline $\begin{array}{l}\text { Academic and professional expert in museum } \\
\text { curatorship }\end{array}$ & $\mathrm{M}$ & 8 & $31-45$ \\
\hline
\end{tabular}

Table 1. Participant Demographics

\begin{tabular}{|c|c|c|c|c|c|c|c|}
\hline Measure & $\begin{array}{c}\text { Strongly } \\
\text { agree }\end{array}$ & Agree & Neutral & Disagree & $\begin{array}{c}\text { Strongly } \\
\text { disagree }\end{array}$ & Mean & $\begin{array}{c}\text { Std. } \\
\text { Dev. }\end{array}$ \\
\hline $\begin{array}{c}\text { I found the headset } \\
\text { comfortable to wear }\end{array}$ & $0.0 \%$ & $77.8 \%$ & $11.1 \%$ & $11.1 \%$ & $0.0 \%$ & 3.67 & .707 \\
\hline $\begin{array}{c}\text { I did not experience } \\
\text { nausea, dizziness, or } \\
\text { headache }\end{array}$ & $77.8 \%$ & $22.2 \%$ & $0.0 \%$ & $0.0 \%$ & $0.0 \%$ & 4.78 & .441 \\
\hline $\begin{array}{c}\text { I could look around the } \\
\text { room comfortably }\end{array}$ & $10.6 \%$ & $44.7 \%$ & $38.3 \%$ & $6.4 \%$ & $0.0 \%$ & 4.44 & 1.014 \\
\hline $\begin{array}{c}\text { I could do air tap on the } \\
\text { virtual objects appropriately }\end{array}$ & $33.3 \%$ & $55.6 \%$ & $11.1 \%$ & $0.0 \%$ & $0.0 \%$ & 4.22 & .667 \\
\hline $\begin{array}{c}\text { I could interact with the } \\
\text { user interface as I }\end{array}$ & $44.4 \%$ & $33.3 \%$ & $11.1 \%$ & $11.1 \%$ & $0.0 \%$ & 4.11 & 1.054 \\
\hline $\begin{array}{c}\text { expected } \\
\text { I could do all functions I }\end{array}$ & $33.3 \%$ & $55.6 \%$ & $11.1 \%$ & $0.0 \%$ & $0.0 \%$ & 4.11 & .928 \\
\hline
\end{tabular}

Table 2. Usability aspects $(1=$ Strongly disagree to $5=$ Strongly agree. $\mathrm{N}=9)$ 
The third question considered prompts and instructions; this area rated positively as users found these instructions helpful. That question was the second highest mean as it resulted in 4.44. There are two comments on this question, the first noted "I needed to think - and be reminded - to look up and down", and the second comment was "I felt I might like to zoom out a bit more".

The fourth inquiry examined the accessibility of the control functions within the application such as 'air tap' to trigger interactions, and it resulted in 4.22 as a mean value. Comments varied between being positive about the ability such as "Yes, after minimal guidance", and other comments were a bit critical such as "It took a bit of practice", and "As for the first time to use it, I need more time to get used of it".

The fifth question examined UX in the new spatial UI which is the primary proposition of this research. Respondents varied between being positive and critical responses to this virtual environment. One participant stated, "I love it, it is much more interactive than I imagined with total freedom of movement". Another expert commented as "Much more interactive than anticipated, loved that you can move around the scene and look in all directions". However, some other comments were critical such as "It is required some time to deal with it".

The sixth question explored the familiarity of the system and how users could progress the information and interact softly according to their desires. Comments mostly were positive as an expert noted: "I started to get used of it very quickly, and the more I use, the better I manage to control the application". Another said, "It is easy to learn after the short tutorial and the way of performing hand air tab". However, an expert has a contradiction with the previous comments as he/she said: "It needs time to get used of it and to understand all the options it has". Generally, the last two questions resulted with similar mean value 4.11.

\subsection{Discussion and Conclusion}

The primary theoretical contributions highlighted in this study derived from previous technical difficulties surrounding the HoloLens FOV in the standard UI as stated in the literature. The evaluation was developed on previous UX principles to enhance the user accessibility aspect of the UI which as standard has a narrow FOV. The test procedure conducted in this study using the prototype HoloLens spatial UI provided substantial evidence to support UX significantly increased with the introduction of a full-screen and content UI. Additionally, this study explains the system structure and developmental pipeline of the Spatial prototype UI for virtual tour guide systems. As this prototype aims to provide an interactive virtual tour guide walking along with a tour with the visitor to unlock visual information as the real human guide do. This system designed to be intractable with a seamless hand air-tap interaction on the spatial UI to provide navigation in the museum and retrieving information on the spot.

The spatial UI prototype gained positive results overall in both usability and accessibility as highlighted in the expert-led questionnaire responses across multiple academic disciplines. The first three questions reviewed the HoloLens ergonomically considering it an essential part of the usability aspects. The weight 
of HoloLens is lighter compared with other MR devices adopted in similar studies (Kerr et al., 2011), as means values reported respectively; 3.79 and 2.13. The weight of the HoloLens is not negatable until the company consider this point in the new generations as the newly released (Allison, 2019). HoloLens users agreed positively on minimal nausea, dizziness, or headache with a high mean value 4.87. Comparing these results with HMD Eyephone LX (Sharples, Cobb, Moody, \& Wilson, 2008), it was reported that $60 \%$ of the users complained from nausea, eyestrain and headache. Users reported being comfortable while looking around the room positively if it compared with another study (Kerr et al., 2011) as means values reported respectively; 4.44 and 1.88. However, comments revealed some confusion concerning the users' vision due to the limited FOV. Experts were able to make the air-tap gestures on the virtual objects positively $($ mean $=4.22)$ comparing to another study (Kerr et al., 2011) with (mean $=3.38$ ). Comments showed a rapid level of familiarity after a short time of demonstrations. This sense of familiarity can indicate potential usage of the headset applications as Wagner et al. (2005) claimed. Experts could interact with the UI as they expect positively $($ mean $=4.11)$ and this represents how the UI is intuitive and usable, which reflects on the user experience eventually. Comparing this UI with other studies, (Kerr et al., 2011) investigated the interaction with UI (mean $=3.38)$. Experts could do all functions designed in the system with $($ mean $=4.11)$, and this can inform how the ease of use enhanced interaction with the designed UI. Comparing this result with other studies, (Kerr et al., 2011) informed that their participants became skilful to do all functions with $($ mean $=4.00)$. Experts' comments represented how they managed to control the application and communicate with the system as needed.

According to Tom Dieck et al. (2016), HoloLens could achieve what museum visitors require in terms of feeling comfortable and not being exposed to health problems such as headaches or nausea. Also, the introduced system that incorporates the spatial UI could achieve what the visitor needs in terms of usability, ease of use and usefulness. Moreover, the visitor has the freedom to navigate using the virtual guide without being distracted which can save time and also give the chance to appreciate the exhibited antiques. The research contributes towards overcoming the limitations of HoloLens FOV with some solutions on the designed UI which can enhance usability and increase the sense of being immersed. This process enhances the user's perception within MR which reflects positively on the sensual gratification of museum visitors according to Rauschnabel (2018).

The theoretical contribution of this study may help future UX developers to overcome the HoloLens FOV problem using the principles outlined in this research. The techniques employed in this study are adaptable across different applications and applied in new design infrastructures to incorporate controls such as floating buttons. The UX model utilised in this study accounts for variable user height modification that may also be implemented in new applications to enhance UX.

The HoloLens permits the development of similar prototype spatial UI with specific MR applications as demonstrated in this study. However, the system is 


\section{Ramy Hammady, Minhua Ma \& Carl Strathearn}

limited, and future versions of the HoloLens should examine the instability in the spatial mapping functionality of the system when a person walks in front of the unit during use. Additionally, research into creating a lighter HMD may reduce the negative feedback regarding the weight and neck strain reported with extensive usage of the HoloLens HMD. This issue may impact the wearers of the HoloLens HMD in a museum setting at the user would be expected to wear the until for a considerable amount of time. Moreover, to create lag free MR environments, consideration is required in the placement of $3 \mathrm{D}$ objects within an area to reduce the amount of visual on screen at the same time to avoid delays in the real-time rendering of objects.

According to Rauschnabel (2018), influencing the sensual gratification of museum visitors can encourage them to adopt and use MR HMD in the future. Accordingly, museums have a chance to incorporate these headsets to enhance the museum experience and improve visitor satisfaction. This approach may encourage HMD companies to provide more headsets in the market due to the high demand. Therefore, this paper seeks to recommend this technology for museum visitors and offer it for public use as the potential of reshaping the museum experience via this headset is strongly achievable.

\subsection{Limitations and Future Work}

The small sample size for the system evaluation is needed to be expanded to include more users from different relevant disciplines. Future studies are required to include a more significant number of museum visitors. This issue of the small sample size in this study is due to limitations regarding gaining the museum's permission to allow system evaluation during visitor hours which disallowed museum visitors. With more time and approved access, scholars could investigate other functions such as the stability of the registered holograms on the physical environment and the occlusion problems that occurs while people are walking around.

Future adaptations to the prototype spatial UI will incorporate voice recognition and text-to-speech functions to engage the user in a greater naturalistic mode of communication. Employing this technique may enhance the UX and adaptability across multi-languages may broaden the accessibility of this function. New builds of the HoloLens may eventually incorporate digital visualisations beyond the spatial UI prototype in a 360-degree spectrum with the user situated as a central pivot to the virtual environment. To further enhance the UX, games will be embedded in the application environment to operate in specific zones. This addition will create greater user interactivity and enrichment by supplying further content. The games will implement a reward system to motivate the exploration of rooms and exhibits by discovering rewards, milestone and additional content. These interactions can be encouraged through the historical narrative of the exhibits and individuals within the museum. 


\section{References}

Adabi, K., Rudy, H., Stern, C. S., Weichman, K., Tepper, O., \& Garfein, E. S. (2017). Optimizing Measurements in Plastic Surgery through Holograms with Microsoft Hololens. Plastic and Reconstructive Surgery Global Open, 5 (9 Suppl).

Allison, C. (2019). Microsoft HoloLens 2: Everything we know so far about the mixed reality headset. Retrieved from https://www.wareable.com/ar/microsoft-hololens-2-release-dateprice-specs-495

Bach, C., \& Scapin, D. L. (2004). Obstacles and perspectives for evaluating mixed reality systems usability. Paper presented at the Acte du Workshop MIXER, IUI-CADUI.

Baker, J. (1986). The role of the environment in marketing services: The consumer perspective. The services challenge: Integrating for competitive advantage, 1(1), 79-84.

Billinghurst, M., Poupyrev, I., Kato, H., \& May, R. (2000). Mixing realities in shared space: An augmented reality interface for collaborative computing. Paper presented at the Multimedia and Expo, 2000. ICME 2000. 2000 IEEE International Conference on.

Blattgerste, J., Strenge, B., Renner, P., Pfeiffer, T., \& Essig, K. (2017). Comparing conventional and augmented reality instructions for manual assembly tasks. Paper presented at the Proceedings of the 10th International Conference on PErvasive Technologies Related to Assistive Environments.

Bordegoni, M., Cugini, U., Caruso, G., Polistina, S. J. I. J. o. I. D., \& Manufacturing. (2009). Mixed prototyping for product assessment: a reference framework. 3(3), 177-187.

Bottino, A. G., García, A. M., \& Occhipinti, E. (2017). Holomuseum: a prototype of interactive exhibition with Mixed Reality glasses HoloLens.

Bowman, D., Kruijff, E., LaViola Jr, J. J., \& Poupyrev, I. P. (2004). 3D User interfaces: theory and practice, CourseSmart eTextbook: Addison-Wesley.

Bowman, D. A., Gabbard, J. L., Hix, D. J. P. T., \& Environments, V. (2002). A survey of usability evaluation in virtual environments: classification and comparison of methods. 11(4), 404424.

Bowman, D. A., \& Hodges, L. F. (1999). Formalizing the design, evaluation, and application of interaction techniques for immersive virtual environments. Journal of Visual Languages \& Computing, 10(1), 37-53.

Bray, M. Z. B. (2018). What is mixed reality? Retrieved from https://docs.microsoft.com/enus/windows/mixed-reality/mixed-reality

Bright, P. (2015). HoloLens: Still magical, but with the ugly taint of reality. Retrieved from https://arstechnica.com/gadgets/2015/05/hololens-still-magical-but-with-the-ugly-taintof-reality/

Brondi, R., Carrozzino, M., Lorenzini, C., \& Tecchia, F. (2016). Using Mixed Reality and Natural interaction in Cultural Heritage Applications. Informatica, 40(3).

Brooke, J. (1996). SUS-A quick and dirty usability scale. Usability evaluation in industry, 189(194), 4-7.

Bulearca, M., \& Tamarjan, D. (2010). Augmented reality: A sustainable marketing tool. Global business and management research: An international journal, 2(2), 237-252.

Charara, P. L. S. (2017). The best smartglasses 2017: Snap, Vuzix, ODG, Sony \& more. Retrieved from https://www.wareable.com/headgear/the-best-smartglasses-google-glass-and-therest

Chuah, S. H.-W. (2018). Why and who will adopt extended reality technology? Literature review, synthesis, and future research agenda. Literature Review, Synthesis, Future Research Agenda.

Coppens, A. (2017). Merging real and virtual worlds: An analysis of the state of the art and practical evaluation of Microsoft Hololens. arXiv preprint arXiv:1706.08096.

Cortana, J. (2017). The Future of Holograms in Museums. Retrieved from https://www.cortinaproductions.com/holograms-in-museums/

DeLaOsa, J. (2017). Ford Designs Next-Gen Cars Using Microsoft's HoloLens. Retrieved from https://www.ecnmag.com/blog/2017/09/ford-designs-next-gen-cars-using-microsoftshololens

Dohse, K. C. (2007). Effects of field of view and stereo graphics on memory in immersive command and control. 
Epson. (2015). Moverio BT-300. Retrieved from https://www.epson.co.uk/products/see-throughmobile-viewer/moverio-bt-300

Evans, G., Miller, J., Pena, M. I., MacAllister, A., \& Winer, E. (2017). Evaluating the Microsoft HoloLens through an augmented reality assembly application. Paper presented at the Degraded Environments: Sensing, Processing, and Display 2017.

Fonnet, A., Alves, N., Sousa, N., Guevara, M., \& Magalhães, L. (2017). Heritage BIM integration with mixed reality for building preventive maintenance. Paper presented at the Computação Gráfica e Interação (EPCGI), $201724^{\circ}$ Encontro Português de.

Hall, T., Ciolfi, L., Bannon, L., Fraser, M., Benford, S., Bowers, J., . . Schnädelbach, H. (2001). The visitor as virtual archaeologist: explorations in mixed reality technology to enhance educational and social interaction in the museum. Paper presented at the Proceedings of the 2001 conference on Virtual reality, archeology, and cultural heritage.

Hix, D., \& Hartson, H. R. J. N. Y. (1993). Developing User Interfaces: Ensuring Usability Through Product \& Process (Wiley).

Hockett, P., \& Ingleby, T. (2016). Augmented reality with HoloLens: Experiential architectures embedded in the real world. arXiv preprint arXiv:1610.04281.

Höllerer, T., Feiner, S., Terauchi, T., Rashid, G., \& Hallaway, D. (1999). Exploring MARS: developing indoor and outdoor user interfaces to a mobile augmented reality system. Computers \& Graphics, 23(6), 779-785.

Hsieh, Y.-T., Jylhä, A., Orso, V., Gamberini, L., \& Jacucci, G. (2016). Designing a willing-to-usein-public hand gestural interaction technique for smart glasses. Paper presented at the Proceedings of the 2016 CHI Conference on Human Factors in Computing Systems.

Hughes, C. E., Smith, E., Stapleton, C., \& Hughes, D. E. (2004). Augmenting museum experiences with mixed reality. Paper presented at the Proceedings of KSCE 2004.

Inc., G. (2014). The Glass Explorer Program. Retrieved from http://www.google.com/glass/start/.

Insight, C. (2016). Wearables Momentum Continues. Retrieved from www.ccsinsight.com: https://www.ccsinsight.com/press/company-news/2516-wearables-momentum-continues

Kalantari, M. (2017). Consumers' adoption of wearable technologies: literature review, synthesis, and future research agenda. International Journal of Technology Marketing, 12(3), 274307.

Kalantari, M., \& Rauschnabel, P. (2018). Exploring the early adopters of augmented reality smart glasses: The case of Microsoft HoloLens. In Augmented Reality and Virtual Reality (pp. 229-245): Springer.

Karoulis, A., Sylaiou, S., \& White, M. (2006). Usability evaluation of a virtual museum interface. Informatica, 17(3), 363-380.

Keighrey, C., Flynn, R., Murray, S., \& Murray, N. (2017). A QoE evaluation of immersive augmented and virtual reality speech \& language assessment applications. Paper presented at the Quality of Multimedia Experience (QoMEX), 2017 Ninth International Conference on.

Kerr, S. J., Rice, M. D., Teo, Y., Wan, M., Cheong, Y. L., Ng, J., . . Wren, D. (2011). Wearable mobile augmented reality: evaluating outdoor user experience. Paper presented at the Proceedings of the 10th International Conference on Virtual Reality Continuum and Its Applications in Industry.

Kress, B. C., \& Cummings, W. J. (2017). 11-1: Invited Paper: Towards the Ultimate Mixed Reality Experience: HoloLens Display Architecture Choices. Paper presented at the SID Symposium Digest of Technical Papers.

Labuschagne, A. (2003). Qualitative research-airy fairy or fundamental? The qualitative report, $8(1)$, $100-103$

LaViola Jr, J. J. (2008). Bringing VR and spatial 3D interaction to the masses through video games. IEEE Computer Graphics and Applications, 28(5).

Leap, M. (2018). Magic Leap. Retrieved from https://www.magicleap.com/

Lee, G. A., Nelles, C., Billinghurst, M., \& Kim, G. J. (2004). Immersive authoring of tangible augmented reality applications. Paper presented at the Proceedings of the 3rd IEEE/ACM international Symposium on Mixed and Augmented Reality.

Lv, Z., Halawani, A., Feng, S., Ur Réhman, S., Li, H. J. P., \& Computing, U. (2015). Touch-less interactive augmented reality game on vision-based wearable device. 19(3-4), 551-567.

Melnick, K. (2017). Art Exhibit Launches Virtual Museum Using Microsoft Hololens. 
Microsoft. (2015). Microsoft HoloLens. Retrieved from https://www.microsoft.com/enus/hololens/buy

Microsoft. (2017). What is MixedRealityToolkit-Unity? Retrieved from https://github.com/Microsoft/MixedRealityToolkit-Unity

Milgram, P., Takemura, H., Utsumi, A., \& Kishino, F. (1994). Augmented Reality: A class of displays on the reality-virtuality continuum. Telemanipulator and Telepresence Technologies, 2351.

Paelke, V., Sester, M. J. I. J. o. P., \& Sensing, R. (2010). Augmented paper maps: Exploring the design space of a mixed reality system. 65(3), 256-265.

Park, S., Chung, K., \& Jayaraman, S. (2014). Wearables: Fundamentals, advancements, and a roadmap for the future. In Wearable sensors (pp. 1-23): Elsevier.

Pollalis, C., Fahnbulleh, W., Tynes, J., \& Shaer, O. (2017). HoloMuse: Enhancing engagement with archaeological artifacts through gesture-based interaction with holograms. Paper presented at the Proceedings of the Tenth International Conference on Tangible, Embedded, and Embodied Interaction.

Prasuethsut, L. (2016). Meta 2 first impressions: AR feels closer than ever. Retrieved from https://www.wareable.com/ar/meta-2-review

Pratt, P., Ives, M., Lawton, G., Simmons, J., Radev, N., Spyropoulou, L., \& Amiras, D. (2018). Through the HoloLens ${ }^{\mathrm{TM}}$ looking glass: augmented reality for extremity reconstruction surgery using 3D vascular models with perforating vessels. European Radiology Experimental, 2(1), 2.

Raptis, G. E., Fidas, C., \& Avouris, N. (2017). Cultural Heritage Gaming: Effects of Human Cognitive Styles on Players' Performance and Visual Behavior. Paper presented at the Adjunct Publication of the 25th Conference on User Modeling, Adaptation and Personalization.

Rauschnabel, P. A. (2018). Virtually enhancing the real world with holograms: An exploration of expected gratifications of using augmented reality smart glasses. Psychology Marketing, $35(8), 557-572$.

Ricci, A., Piunti, M., Tummolini, L., \& Castelfranchi, C. J. I. P. C. (2015). The mirror world: Preparing for mixed-reality living. 14(2), 60-63.

Sauer, S., \& Göbel, S. (2003). Dinohunter: Game based learn experience in Museums. Paper presented at the ICHIM'03.

Sharples, S., Cobb, S., Moody, A., \& Wilson, J. R. J. D. (2008). Virtual reality induced symptoms and effects (VRISE): Comparison of head mounted display (HMD), desktop and projection display systems. 29(2), 58-69.

Shneiderman, B., \& Plaisant, C. (1992). De signing the User Interface. In Strategies for Effective Human Computer Interaction: Addison-Wesley Publishing Company USA.

Spreer, P., \& Kallweit, K. (2014). Augmented reality in retail: assessing the acceptance and potential for multimedia product presentation at the PoS. Transactions on Marketing Research, 1(1), 20-35.

Stearns, L., DeSouza, V., Yin, J., Findlater, L., \& Froehlich, J. E. (2017). Augmented Reality Magnification for Low Vision Users with the Microsoft Hololens and a Finger-Worn Camera. Paper presented at the Proceedings of the 19th International ACM SIGACCESS Conference on Computers and Accessibility.

Syed, A., Zakaria, A., \& Lozanoff, S. (2017). dark Room To Augmented Reality: Application Of Hololens Technology For Oral Radiological Diagnosis. Oral surgery, oral medicine, oral pathology and oral radiology, 124(1), e33.

Szinte, M., \& Cavanagh, P. J. P. o. (2012). Apparent motion from outside the visual field, retinotopic cortices may register extra-retinal positions. 7(10), e47386.

Tom Dieck, M. C., Jung, T., Han, D.-I. J. J. o. H., \& Technology, T. (2016). Mapping requirements for the wearable smart glasses augmented reality museum application. 7(3), 230-253.

Wagner, D., Pintaric, T., Ledermann, F., \& Schmalstieg, D. (2005). Towards massively multi-user augmented reality on handheld devices. Paper presented at the International Conference on Pervasive Computing.

Wang, W., Wu, X., Chen, G., \& Chen, Z. (2018). Holo3DGIS: Leveraging Microsoft HoloLens in 3D Geographic Information. ISPRS International Journal of Geo-Information, 7(2), 60. 
Ramy Hammady, Minhua Ma \& Carl Strathearn

Yang, H., Yu, J., Zo, H., \& Choi, M. (2016). User acceptance of wearable devices: An extended perspective of perceived value. Telematics and Informatics, 33(2), 256-269.

Zeller, M., Baker, K., \& Bray, B. (2018a). Spatial mapping. In M. s. c. a. room (Ed.), (Vol. 640px × 360px). Microsoft Holography Academy.

Zeller, M., Baker, K., \& Bray, B. (2018b). Spatial mapping. Retrieved from https://docs.microsoft.com/en-us/windows/mixed-reality/spatial-mapping

Zhang, L., Dong, H., \& El Saddik, A. Towards a QoE Model to Evaluate Holographic Augmented Reality Devices. 